\title{
Tracing metabolism from lignocellulosic biomass and gaseous substrates to products with stable-isotopes
}

\author{
Jacqueline E. Gonzalez, Maciek R. Antoniewicz ${ }^{*}$ \\ Department of Chemical and Biomolecular Engineering, Metabolic Engineering and Systems \\ Biology Laboratory, University of Delaware, Newark DE 19716, USA
}

*Corresponding author:

Maciek R. Antoniewicz

Department of Chemical and Biomolecular Engineering

University of Delaware

150 Academy St

Newark, DE 19716

Tel.: 302-831-8960

Fax.: 302-831-1048

Email:mranton@udel.edu 


\begin{abstract}
Bioengineered microbes offer a practical and sustainable alternative to traditional industrial approaches. To increase the economic feasibility of biological processes, microbial isolates are engineered to take up inexpensive feedstocks (including lignocellulosic biomass, syngas, methane, and carbon dioxide), and convert them into substrates of central metabolism and further into value-added products. To trace the metabolism of these feedstocks into products, isotopic tracers are applied together with isotopomer analysis techniques such as ${ }^{13} \mathrm{C}$-metabolic flux analysis to provide a detailed picture of pathway utilization. Flux data is then integrated with kinetic models and constraint-based approaches to identify metabolic bottlenecks, propose novel metabolic engineering strategies, and improve process performance.
\end{abstract}

\title{
Introduction
}

The production of chemicals through bioconversion has received much attention over the past decade [1]. Focus is now shifting towards the utilization of cheap, renewable bio-feedstocks, ultimately leading to reduced operating costs, reduced reliance on non-renewable feedstocks and feedstocks that compete with food supply, and reduced environmental impact $[2,3]$. Renewable feedstocks include lignocellulosic biomass and gaseous substrates such as syngas, methane, hydrogen gas, and carbon dioxide. The primary renewable feedstock, lignocellulosic biomass, is composed of three main components, two of which, cellulose and hemicellulose, can be hydrolyzed to C6 and C5 sugar monomers and used as substrates in fermentations. Alternatively, biomass can be gasified to produce syngas, a mixture of carbon dioxide, carbon monoxide and hydrogen, for use in mixotrophic fermentations [4,5]. Moreover, biological processes such as anaerobic digesters produce large quantities of methane, yet another potential source of carbon 
and energy. With the availability of these renewable feedstocks, metabolic engineering efforts are geared towards engineering organisms to utilize one or more of these substrates and produce value-added chemicals. These efforts have consisted of introducing new metabolic capabilities into model organisms such as E. coli and $S$. cerevisiae, and engineering promising new organisms capable of metabolizing these feedstocks at higher rates and yields $[3,6]$.

With the wide range of potential substrates and products, it is of critical importance to evaluate the economic feasibility of bioprocesses, determine the capabilities of microbial systems, identify targets for improvements, and select ideal candidates for industrial implementation. Significant efforts have been directed at understanding cellular metabolism of microbes and identifying key regulatory mechanisms and potential kinetic limitations. A powerful method for characterizing in vivo metabolism is through the use of ${ }^{13} \mathrm{C}$-labeled substrates (or ${ }^{13} \mathrm{C}$-tracers). Tracing techniques allow quantitative evaluation of the flow of carbon from feedstocks to central metabolism and further into the desired products. Additionally, advanced techniques such as ${ }^{13} \mathrm{C}$ metaboic flux analysis $\left({ }^{13} \mathrm{C}-\mathrm{MFA}\right)$ can be applied to gain a fine-grained picture of native metabolism and metabolic changes that result from genetic manipulations. In this contribution, we provide an overview of the wide range of pathways that are being implemented to convert renewable substrates into value-added products, and discuss methods for elucidating metabolism through these pathways using advanced ${ }^{13} \mathrm{C}$-tracing techniques.

\section{Metabolism of lignocellulosic biomass}

The major costs in biological processes are substrate-related [7]. Thus, organisms which can utilize a broad spectrum of renewable substrates offer a major competitive advantage in the 
development of technologies for the production of next-generation fuels and chemicals. The primary renewable feedstock, lignocellulosic biomass, is composed of cellulose, hemicellulose, and lignin. Cellulose is a polymer of glucose, while hemicellulose is composed of both C6 (glucose, mannose, galactose) and C5 (xylose, arabinose) sugars. Biological conversion of these sugars has received much attention. Metabolism of sugars starts with transport of the sugar into the cells followed by activation (phosphorylation). The main mechanisms for sugar transport are active transport (via the PTS transport system, or ATP-dependent ABC transporters), and passive transport by permeases. For example, in E. coli, glucose is transported and phosphorylated simultaneously via the PTS system at the expense of one ATP equivalent, while two ATPs are expended when xylose serves as the carbon source, i.e. one for transport (high-affinity $\mathrm{ABC}$ transporter) and the second for activation (phosphorylation). Other sugars, e.g. lactose, are transported by permeases. The two classical pathways for sugar catabolism are glycolysis and pentose phosphate pathway (Figure 1). Hexoses feed directly into glycolysis at glucose 6phosphate (G6P) or fructose 6-sphosphate (F6P), while pentoses feed into the pentose phosphate pathway at xylulose 5-phosphate (X5P). Many organisms are capable of catabolizing more than one sugar; however, in most cases carbon catabolite repression (CCR) results in preferential utilization of certain sugars over others.

Various engineering strategies have been employed to eliminate CCR, often resulting in more efficient bioprocesses. A successful strategy has been knocking out components of the PTS system and applying adaptive evolution to improve sugar co-utilization. For example, BalderasHernandez et al. adaptively evolved E. coli lacking a functional PTS system under anaerobic conditions to achieve a glucose/xylose co-utilizing E. coli strain [8]. Adaptive evolution was also 
successfully applied to generate an improved strain of the thermophile Thermus thermophilus that efficiently co-utilized glucose and xylose without CCR [9]. In another study, the pts $G$ gene was removed in E. coli to relieve CCR and allowed production of 3-hydroxypropionic acid from both glucose and xylose in fed-batch fermentations [10]. The model anaerobic organism Clostridium acetobutylicum was also evaluated for sugar co-utilization [11]. Analysis of CCR genes in C. acetobutylicum identified targets for engineering simultaneous utilization of glucose and xylose [12]. When glucose repression of xylose catabolism was removed, product titers from co-utilization of both substrates were comparable to wild-type titers [13].

\section{Native and synthetic pathways for conversion of renewable feedstocks}

Glycolysis and pentose phosphate pathway are the main routes for sugar catabolism in microbes; however, these pathways result in significant loss of carbon $\left(\mathrm{CO}_{2}\right.$ released), which reduces theoretical yields [5]. Recent metabolic engineering strategies have focused on integrating alternative catabolic pathways into host organisms with the goal of producing more efficient bioconversions (Table 1). Figure 2 provides an overview of the wide range of alternative metabolic pathways that are being considered for conversion of renewable feedstocks. A classic alternative to the glycolysis pathway is the Entner-Doudoroff pathway, which produces glyceraldehyde phosphate (GAP) and pyruvate, as well as the reducing cofactor NADPH. A less common variant of the ED pathway is the non-phosphorylated ED pathway which produces pyruvate and glycerladehyde and does not involve phosphorylated intermediates. This pathway can connect to the classic ED pathway through the activity of 2-keto-3-deoxygluconate kinase, thus forming the semi-phosphorylated ED pathway. Studies have revealed presence of these alternative ED pathways in certain thermophilic and halophilic archaea [14]. 
Another route to bypass glycolysis and reach useful intermediates without carbon loss is by employing phosphoketolases [15]. Phosphoketolases are enzymes that are distantly related to transketolases and are found natively in certain Lactobacillus, Bifidobacterium and Clostridium species [11,16-18]. These promiscuous enzymes can cleave either F6P or X5P to acetylphosphate and a corresponding sugar phosphate, erythrose-4-phosphate (E4P) and GAP, respectively. The sugar phosphates can then be recycled back to F6P or X5P. It was demonstrated that by employing phosphoketolases, sugars can be converted to acetate and other products at near-theoretical yields, as first described in [19] and later in [20] (where it was rebranded "non-oxidative glycolysis", NOG). Phosphoketolases have been successfully incorporated into E. coli and S. cerevisiae for acetone, ethanol and fatty acid production [21-23].

Other promising routes for sugar metabolism include the Weimberg and Dahms pathways and a new synthetic xylulose 1-phosphate pathway [24]. The Weimberg pathway bypasses glycolysis to reach the TCA cycle intermediate $\alpha$-ketoglutarate, while the Dahms pathway bypasses glycolysis to produce pyruvate and glycoaldehyde. These conversions occur without phosphorylation of intermediates or carbon loss, which results in increased maximum theoretical yields of products such as itaconic acid, succinic acid, and glutamic acid. The Weimberg pathway was successfully introduced into $E$. coli and $C$. glutamicum for more efficient conversion of xylose and arabinose into various products [25-27]. This pathway is also being investigated for industrial purposes in solvent-tolerant organisms that natively express this pathway, such as Pseudomonas taiwanensis [28]. Recently, an alternative xylose degradation pathway was engineered, termed the xylulose 1-phosphate (X1P) pathway [24]. In X1P pathway, 
the first two carbon atoms of xylose are converted to glycoaldehyde from which products such as ethylene glycol and glycolic acid can be produced [29,30]. Glycoladehyde can also enter the TCA cycle via the glyoxylate shunt after being converted to glyoxylate.

Renewable gases are also increasingly evaluated as potential co-substrates for the production of chemicals [31,32]. These gases include carbon dioxide, carbon monoxide, and hydrogen gas derived from biomass gasification, and methane derived from anaerobic digestion of organic wastes. Multiple avenues are implemented to increase the ability of microorganisms to utilize gaseous substrates. Two well studied carbon fixation pathways are the Calvin cycle and the Wood-Ljungdahl pathway [33]. The Calvin cycle fixes $\mathrm{CO}_{2}$ to the $\mathrm{C} 5$ sugar ribulose bisphosphate (RuBP) forming two molecules of 3-phosphoglycerate (3PG), catalyzed by ribulose1,5-bisphosphate carboxylase-oxygenase (RubisCO), an enzyme that is essential in photosynthesis. While the Calvin cycle is most prevalent in photosynthetic organisms, it is also found in some bacteria such as the Sulfobacillus and Oscillochloris species [34]. Compared to other carbon fixation pathways, however, the Calvin cycle is more energy demanding as it consumes seven ATP to form one molecule of pyruvate [33]. Regardless, there have been many reports of using autotrophic organisms to produce chemicals such as isopropanol, isoprene, and sugars from $\mathrm{CO}_{2}$ [35]. There is also the potential to introduce this pathway into non-carbon fixing organisms, which could effectively increase product yields and decrease carbon loss [3638]. In a recent study, it was demonstrated that expression of the Calvin cycle in S. cerevisiae resulted in higher ethanol yields and eliminated glycerol by-product formation [39]. 
In addition to the Calvin cycle, the Wood-Ljungdahl (WL) pathway is a widely studied carbon fixation route that can utilize both $\mathrm{CO}_{2}$ and $\mathrm{CO}$ as substrates $[4,40]$. In WL pathway, one molecule of $\mathrm{CO}_{2}$ is converted to $\mathrm{CO}$ by carbon monoxide dehydrogenase, and a separate cascade of steps forms a methyl group from $\mathrm{CO}_{2}$. The enzyme acetyl-CoA synthase then brings the $\mathrm{CO}$ and methyl group together to form AcCoA. Depending on the carbon source, electrons can be obtained from $\mathrm{H}_{2}$ or $\mathrm{CO}$. The WL pathway is energetically more efficient than the Calvin cycle and is found exclusively in anaerobic organisms $[5,41]$.

Methane is another potential substrate that can be converted into fuels and chemicals $[42,43]$. Methane is first converted to methanol and then to formaldehyde, which gives the cell access to several carbon assimilation pathways. Two of these pathways are depicted in Figure 2, the ribulose monophosphate (RuMP) pathway and the xylulose monosphate (XuMP) pathway. The RuMP pathway uses two enzymes to fix formaldehyde to ribulose 5-phosphate (Ru5P) and enter central carbon metabolism as F6P. Similarly, the XuMP pathway attaches formaldehyde to a C5 sugar, X5P, and forms GAP and dihydroxyacetone phosphate (DHAP), which are intermediates of glycolysis. The RuMP pathway is found in methylotrophic bacteria such as Mycobacterium gastri and Bacillus methanolicus, while the XuMP pathway is found natively in methylotrophic yeasts. Methanol is an attractive substrate because it contains more electrons per carbon than sugars; thus, theoretical yields of reduced products, including biofuels, are higher with methanol as substrate. So far, it has been difficult to generate a non-native fully methylotrophic microbe [44], while engineering co-utilization of methanol and sugars has been more successful. For example, C. glutamicum was engineered to consume methanol and ribose and produce 
cadaverine [45], and Pichia pastoris was shown to produce recombinant proteins from methanol and glucose [46].

\section{Tracing metabolism with isotopic tracers}

Tracing how substrates are utilized and converted into products is critical in assessing the efficiency of engineered pathways and to guide further metabolic engineering strategies. Stableisotope labeling techniques (primarily with ${ }^{13} \mathrm{C}$-tracers) are widely used in metabolic engineering to quantify carbon flux for this purpose $[47,48]$. In tracer experiments, an isotopically labeled substrate is added to the culture, e.g. $\left[\mathrm{U}_{-}{ }^{13} \mathrm{C}\right]$ glucose, resulting in the incorporation of ${ }^{13} \mathrm{C}$ atoms into intermediates of cellular metabolism and eventually into products. By quantifying fractional ${ }^{13} \mathrm{C}$-labeling of various metabolites (products) the contribution of a particular substrate can be determined. Tracer experiments are especially useful when multiple substrates are present and when complex additives such as yeast extract are used in fermentations. By measuring ${ }^{13} \mathrm{C}$ labeling over time it is also possible to elucidate relative rates of substrate utilization. For example, a constant isotopic labeling indicates that substrates are consumed simultaneously [5]. On the other hand, if labeling changes in time, then the rate at which the labeling changes can be translated into relative flux changes [49].

Tracing techniques require accurate and sensitive analytical approaches for measuring isotopic labeling. Currently, mass spectrometry (MS) based techniques are preferred due to their high sensitivity and the potential to obtain positional labeling information through tandem mass spectrometry [50]. MS-based approaches require a relatively small sample size and give access to intracellular metabolites. Figure 3 provides an overview of metabolites in central carbon 
metabolism that can be measured with GC-MS and LC-MS/MS. While there is significant overlap in the range of metabolites that can be measured with these two approaches, LC-MS/MS is generally more suitable for measuring phosphorylated metabolites in the pentose phosphate pathway, nucleosides and other large molecules [51], while GC-MS is preferred for measuring labeling of amino acids, carbohydrates, and TCA cycle metabolites, given that GC-MS produces multiple fragments that yields additional labeling information [52,53]. Advanced GC-MS/MS approaches have also been developed for more complete analysis of labeling patterns [54]. Ultimately, the choice of the measurement technique depends on the information desired, the cost of performing labeling measurements, and available resources.

\section{Measuring metabolism with ${ }^{13} \mathrm{C}$ metabolic flux analysis}

In order for biological processes to be economically viable, substrates must be converted to products at near-theoretical yields [7]. This generally requires significant rewiring of cellular metabolism. Compared to native metabolism, which has evolved to optimize cell growth, optimal product formation will require a different balance of pathway activities to maximize the flow of carbons and electrons from feedstocks to products. ${ }^{13} \mathrm{C}$-Metabolic flux analysis $\left({ }^{13} \mathrm{C}\right.$ MFA) is the best approach to gain quantitative insights into cellular metabolism [48]. Knowledge of metabolic fluxes is important to identify bottlenecks in metabolism and determine specific changes in pathway utilization resulting from genetic manipulations [55]. Experimentally validated fluxes are also used in constraint-based modeling approaches, and fluxes are critical for parameterizing kinetic models of metabolism [56], which are increasingly applied for analyzing metabolic pathways and predicting the outcomes of metabolic engineering interventions given the poor performance of traditional constraint-based approaches [57]. 
Methods for ${ }^{13} \mathrm{C}$-MFA have advanced significantly in the past few years [48]. With current stateof-the-art approaches, it is possible to measure intracellular fluxes with a precision of about 13\% [58]. The ability to generate high-resolution flux maps presents unprecedented opportunities to gain a much more detailed understanding of the regulation of metabolic pathways and in vivo enzyme kinetics. Current best approaches for flux estimation are based on the concept of parallel labeling experiments and integrated ${ }^{13} \mathrm{C}-\mathrm{MFA}$ (Figure 4) [59]. A good example of the power of parallel labeling experiments is the study by Crown et al. [58], where 14 parallel labeling experiments were successfully integrated to generate a detailed flux map for E. coli. Parallel labeling experiments were also successfully applied for analysis of Clostridium acetobutylicum [60], Geobacillus spp. [61], and various mammalian systems [62-65].

The design of optimal labeling experiments is a critical step in ${ }^{13} \mathrm{C}-\mathrm{MFA}$, which until recently did not receive the attention it deserves. It is now well recognized that traditional tracers such as $\left[1-{ }^{13} \mathrm{C}\right]$ glucose and $\left[\mathrm{U}_{-}{ }^{13} \mathrm{C}\right]$ glucose, although relatively cheap, are suboptimal for quantifying precise metabolic fluxes [66]. For example, it was demonstrated that doubly labeled tracers such as $\left[1,2-{ }^{13} \mathrm{C}\right]$ glucose and $\left[1,6-{ }^{13} \mathrm{C}\right]$ glucose are more optimal for ${ }^{13} \mathrm{C}-\mathrm{MFA}$, since these tracers produce fluxes with much smaller confidence intervals [67]. For xylose, optimal tracers include $\left[1,2-{ }^{13} \mathrm{C}\right] \mathrm{xylose}$ and $\left[5-{ }^{13} \mathrm{C}\right] \mathrm{xylose}[61]$.

The choice of tracers becomes even more critical when multiple substrates are present. When studying glucose and xylose co-utilization, the combination of $\left[1-{ }^{13} \mathrm{C}\right]$ glucose and unlabeled xylose has been commonly used (e.g. to elucidate pentose phosphate pathway fluxes in a creA- 
mutant of Aspergillus nidulans [68], and to study the effect of a modified redox pathway in $S$. cerevisiae [69]). A limitation of this tracer scheme is, however, that it only works well when the labeled substrate (in this case glucose) is the main carbon source. If xylose becomes the main carbon source, then ${ }^{13} \mathrm{C}$-labeling becomes dramatically reduced and flux estimation fails. It is therefore advantageous to design labeling experiments that are less dependent on specific fluxes. Logically, it follows that both substrates should be labeled. A number of optimized tracer schemes have been proposed and successfully applied, including $\left[1,2-{ }^{13} \mathrm{C}\right]$ glucose $+[1,2-$ $\left.{ }^{13} \mathrm{C}\right] \mathrm{xylose}$ to elucidate glucose and xylose co-utilization in E. coli [70], and $\left[1,6{ }^{13} \mathrm{C}\right] \mathrm{glucose}+$ $\left[5-{ }^{13} \mathrm{C}\right] \mathrm{xylose}$ for flux analysis in T. thermophilus [9].

Metabolic fluxes from other lignocellulosic sugars have also been studied. For example, Fonseca et al. used [2- $\left.{ }^{13} \mathrm{C}\right]$ arabinose to study arabitol production in arabinose-utilizing yeast strains [71], and Sund et al. used $\left[1-{ }^{13} \mathrm{C}\right]$ glucose, $\left[1-{ }^{13} \mathrm{C}\right] \mathrm{xylose}$, and $\left[1-{ }^{13} \mathrm{C}\right]$ arabinose to estimate phosphoketolase fluxes in Clostridium acetobutylicum [16]. Similarly, Fendt and Sauer used ${ }^{13} \mathrm{C}$ glucose, ${ }^{13} \mathrm{C}$-galactose, and ${ }^{13} \mathrm{C}$-mannose tracers to elucidate sugar metabolism of $\mathrm{S}$. cerevisiae [72]. Earlier studies with Fibrobacter also focused on elucidating catabolism of more complex substrates, including cellulose and cellobiose [73]. By examining the dilution of $\left[1-{ }^{13} \mathrm{C}\right]$ glucose and quantifying the labeling of products during growth on cellulose or cellobiose, the authors were able to determine how glucose was utilized in the presence of cellulose.

\section{Conclusions}


Combining isotopic tracers and ${ }^{13} \mathrm{C}$-metabolic flux analysis is a powerful approach for studying metabolism of engineered microorganisms. This method is especially useful when exploring new pathways for the conversion of non-traditional feedstocks. As highlighted here, there have been extensive efforts in engineering both upstream and downstream metabolism for production of fuels and chemicals. Substrate utilization pathways have been targeted to bestow the ability to consume new carbon sources in industrially viable organisms, and new pathways have been engineered for production of value-added chemicals by bypassing conventional metabolic routes, reducing carbon loss, and improving yield. Given the demonstrated advantages of using both organic (sugars) and inorganic $\left(\mathrm{CO}_{2}, \mathrm{CO}, \mathrm{H}_{2}, \mathrm{CH}_{4}\right)$ feedstocks, it is likely that co-utilization of mixed substrates by mixotrophy will remain an active area of research in the foreseeable future.

\section{Acknowledgements}

This work was supported by the U.S. Department of Energy ARPA-E grant DE-AR0000432. 


\section{TABLES}

Table 1. Examples of native and synthetic pathways for bio-conversion of renewable feedstocks.

\begin{tabular}{|l|l|l|l|}
\hline Metabolic pathway & E. coli & S. cerevisiae & Other organisms \\
\hline Entner-Doudoroff pathway & Native & {$[74]$} & {$[75]$} \\
\hline Wood-Ljungdahl pathway & - & - & {$[5]$} \\
\hline Phosphoketolase pathways & {$[20,21]$} & {$[22,23]$} & {$[11,16,76-78]$} \\
\hline Calvin cycle & {$[36-38]$} & {$[39]$} & {$[79]$} \\
\hline Methylotrophic pathways & {$[44]$} & - & {$[46,80-82]$} \\
\hline Weimberg and Dahms pathways & {$[25,26]$} & - & {$[27,28]$} \\
\hline Other synthetic pathways & {$[24,83]$} & - & - \\
\hline
\end{tabular}

\section{FIGURE LEGENDS}

Figure 1. Conversion of lignocellulose-derived sugars into substrates of central carbon metabolism.

Figure 2. Native and synthetic metabolic pathways for conversion of renewable feedstocks into value-added products.

Figure 3. A) Example of an isotopic labeling experiment with multiple ${ }^{13} \mathrm{C}$-tracers to quantify metabolism of glucose and xylose. B) Overview of mass spectrometry based measurements of metabolism in core metabolic pathways (here, glycolysis and pentose phosphate pathway) for analysis of isotopic labeling and ${ }^{13} \mathrm{C}$-metabolic flux analysis.

Figure 4. Comparison of different designs of isotopic labeling experiments to quantify coutilization of multiple substrates using classical ${ }^{13} \mathrm{C}$-MFA (left), and integrated ${ }^{13} \mathrm{C}$-MFA based on parallel labeling experiments (right). 


\section{REFERENCES}

Papers of special interest $(*)$

Papers of outstanding interest $(* *)$

1. Himmel ME, Bayer EA: Lignocellulose conversion to biofuels: current challenges, global perspectives. Curr Opin Biotechnol 2009, 20:316-317.

2. Elkins JG, Raman B, Keller M: Engineered microbial systems for enhanced conversion of lignocellulosic biomass. Curr Opin Biotechnol 2010, 21:657-662.

3. Liao JC, Mi L, Pontrelli S, Luo S: Fuelling the future: microbial engineering for the production of sustainable biofuels. Nat Rev Microbiol 2016, 14:288-304.

4. Munasinghe PC, Khanal SK: Biomass-derived syngas fermentation into biofuels: Opportunities and challenges. Bioresour Technol 2010, 101:5013-5022.

**5. Jones SW, Fast AG, Carlson ED, Wiedel CA, Au J, Antoniewicz MR, Papoutsakis ET, Tracy BP: CO2 fixation by anaerobic non-photosynthetic mixotrophy for improved carbon conversion. Nat Commun 2016:7:12800 doi: 12810.11038/ncomms12800.

This study demonstrates that mixotrophic fermentations of sugars and gasses can improve product yields. In this case Clostridium ljungdahlii was engineered to produce acetone with a mass yield that corresponds to $138 \%$ of the theoretical heterotrophic maximum yield.

*6. Cordova LT, Long CP, Venkataramanan KP, Antoniewicz MR: Complete genome sequence, metabolic model construction and phenotypic characterization of Geobacillus LC300, an extremely thermophilic, fast growing, xylose-utilizing bacterium. Metab Eng 2015, 32:74-81.

This paper describes a new extremely thermophilic strain that catabolizes xylose, glucose and other sugars at rates that are several-fold higher than the best engineered mesophilic organisms.

7. Papoutsakis ET: Reassessing the progress in the production of advanced biofuels in the current competitive environment and beyond: what are the successes and where progress eludes us and why. Ind Eng Chem Res 2015, 54:10170-10182.

8. Balderas-Hernandez VE, Hernandez-Montalvo V, Bolivar F, Gosset G, Martinez A: Adaptive evolution of Escherichia coli inactivated in the phosphotransferase system operon improves co-utilization of xylose and glucose under anaerobic conditions. Appl Biochem Biotechnol 2011, 163:485-496.

*9. Cordova LT, Lu J, Cipolla RM, Sandoval NR, Long CP, Antoniewicz MR: Co-utilization of glucose and xylose by evolved Thermus thermophilus LC113 strain elucidated by 13C metabolic flux analysis and whole genome sequencing. Metab Eng 2016, 37:6371. 
This paper describes the first rigorous ${ }^{13} \mathrm{C}$-metabolic flux analysis study of glucose/xylose co-utilization. Adaptive laboratory evolution was successfully applied to generate a strain that consumes multiple substrates without carbon catabolite repression.

10. Jung IY, Lee JW, Min WK, Park YC, Seo JH: Simultaneous conversion of glucose and xylose to 3-hydroxypropionic acid in engineered Escherichia coli by modulation of sugar transport and glycerol synthesis. Bioresour Technol 2015, 198:709-716.

11. Aristilde L, Lewis IA, Park JO, Rabinowitz JD: Hierarchy in pentose sugar metabolism in Clostridium acetobutylicum. Appl Environ Microbiol 2015, 81:1452-1462.

12. Grimmler C, Held C, Liebl W, Ehrenreich A: Transcriptional analysis of catabolite repression in Clostridium acetobutylicum growing on mixtures of D-glucose and Dxylose. J Biotechnol 2010, 150:315-323.

13. Ren C, Gu Y, Hu S, Wu Y, Wang P, Yang Y, Yang C, Yang S, Jiang W: Identification and inactivation of pleiotropic regulator CcpA to eliminate glucose repression of xylose utilization in Clostridium acetobutylicum. Metab Eng 2010, 12:446-454.

14. Siebers B, Schonheit P: Unusual pathways and enzymes of central carbohydrate metabolism in Archaea. Curr Opin Microbiol 2005, 8:695-705.

15. Henard CA, Freed EF, Guarnieri MT: Phosphoketolase pathway engineering for carbonefficient biocatalysis. Curr Opin Biotechnol 2015, 36:183-188.

16. Sund CJ, Liu S, Germane KL, Servinsky MD, Gerlach ES, Hurley MM: Phosphoketolase flux in Clostridium acetobutylicum during growth on L-arabinose. Microbiology 2015, 161:430-440.

17. Liu L, Zhang L, Tang W, Gu Y, Hua Q, Yang S, Jiang W, Yang C: Phosphoketolase pathway for xylose catabolism in Clostridium acetobutylicum revealed by $13 \mathrm{C}$ metabolic flux analysis. J Bacteriol 2012, 194:5413-5422.

18. Xiong W, Lee TC, Rommelfanger S, Gjersing E, Cano M, Maness PC, Ghirardi M, Yu J: Phosphoketolase pathway contributes to carbon metabolism in cyanobacteria. Nat Plants 2015, 2:15187.

19. Chinen A, Kozlov YI, Hara Y, Izui H, Yasueda H: Innovative metabolic pathway design for efficient l-glutamate production by suppressing $\mathrm{CO} 2$ emission. J Biosci Bioeng 2007, 103:262-269.

20. Bogorad IW, Lin TS, Liao JC: Synthetic non-oxidative glycolysis enables complete carbon conservation. Nature 2013, 502:693-697.

21. Yang X, Yuan Q, Zheng Y, Ma H, Chen T, Zhao X: An engineered non-oxidative glycolysis pathway for acetone production in Escherichia coli. Biotechnol Lett 2016, 38:1359-1365.

22. Sonderegger M, Schumperli M, Sauer U: Metabolic engineering of a phosphoketolase pathway for pentose catabolism in Saccharomyces cerevisiae. Appl Environ Microbiol 2004, 70:2892-2897.

23. de Jong BW, Shi S, Siewers V, Nielsen J: Improved production of fatty acid ethyl esters in Saccharomyces cerevisiae through up-regulation of the ethanol degradation pathway and expression of the heterologous phosphoketolase pathway. Microb Cell Fact 2014, 13:39.

**24. Cam Y, Alkim C, Trichez D, Trebosc V, Vax A, Bartolo F, Besse P, Francois JM, Walther T: Engineering of a Synthetic Metabolic Pathway for the Assimilation of (d)-Xylose into Value-Added Chemicals. ACS Synth Biol 2016, 5:607-618. 
In this work a novel metabolic pathway was engineered in E. coli to convert xylose into products including ethylene glycol and glycolic acid at high yields.

**25. Choi SY, Park SJ, Kim WJ, Yang JE, Lee H, Shin J, Lee SY: One-step fermentative production of poly(lactate-co-glycolate) from carbohydrates in Escherichia coli. Nat Biotechnol 2016, 34:435-440.

In this work $E$. coli was engineered to produce a wide range of biodegradable and biocompatible synthetic polymers from glucose and xylose. This was achieved by introducing the Dahms pathway to produce glycolate from xylose, and by eliminating carbon catabolite repression.

*26. Tai YS, Xiong M, Jambunathan P, Wang J, Wang J, Stapleton C, Zhang K: Engineering nonphosphorylative metabolism to generate lignocellulose-derived products. $\mathrm{Nat}$ Chem Biol 2016, 12:247-253.

In this work the authors introduced the Weimberg pathway into E. coli and constructed an artificial biosynthetic pathways to 1,4-butanediol (BDO) that could achieve a high theoretical molar yield from D-xylose, L-arabinose and D-galacturonate. The titers, rates and yields were higher than those previously reported using conventional pathways.

27. Radek A, Krumbach K, Gatgens J, Wendisch VF, Wiechert W, Bott M, Noack S, Marienhagen J: Engineering of Corynebacterium glutamicum for minimized carbon loss during utilization of D-xylose containing substrates. J Biotechnol 2014, 192 Pt A:156-160.

28. Kohler KA, Blank LM, Frick O, Schmid A: D-Xylose assimilation via the Weimberg pathway by solvent-tolerant Pseudomonas taiwanensis VLB120. Environ Microbiol 2015, 17:156-170.

29. Pereira B, Li ZJ, De Mey M, Lim CG, Zhang H, Hoeltgen C, Stephanopoulos G: Efficient utilization of pentoses for bioproduction of the renewable two-carbon compounds ethylene glycol and glycolate. Metab Eng 2016, 34:80-87.

30. Pereira B, Zhang H, De Mey M, Lim CG, Li ZJ, Stephanopoulos G: Engineering a novel biosynthetic pathway in Escherichia coli for production of renewable ethylene glycol. Biotechnol Bioeng 2016, 113:376-383.

31. Fast AG, Schmidt ED, Jones SW, Tracy BP: Acetogenic mixotrophy: novel options for yield improvement in biofuels and biochemicals production. Curr Opin Biotechnol 2015, 33:60-72.

32. Hu P, Chakraborty S, Kumar A, Woolston B, Liu H, Emerson D, Stephanopoulos G: Integrated bioprocess for conversion of gaseous substrates to liquids. Proc Natl Acad Sci U S A 2016, 113:3773-3778.

33. Fast AG, Papoutsakis ET: Stoichiometric and energetic analyses of non-photosynthetic CO2-fixation pathways to support synthetic biology strategies for production of fuels and chemicals. Curr Opin Chem Eng 2012, 1:380-395.

34. Berg IA, Kockelkorn D, Ramos-Vera WH, Say RF, Zarzycki J, Hugler M, Alber BE, Fuchs G: Autotrophic carbon fixation in archaea. Nat Rev Microbiol 2010, 8:447-460. 
35. Ducat DC, Way JC, Silver PA: Engineering cyanobacteria to generate high-value products. Trends Biotechnol 2011, 29:95-103.

**36. Antonovsky N, Gleizer S, Noor E, Zohar Y, Herz E, Barenholz U, Zelcbuch L, Amram S, Wides A, Tepper N, et al.: Sugar Synthesis from CO2 in Escherichia coli. Cell 2016, 166:115-125.

This work describes functional expression of the Calvin cycle in E. coli. This is the first example of a non-native carbon assimilation cycle in a heterotrophic organism in which all of the pathway intermediates and products are synthesized solely from $\mathrm{CO}_{2}$.

37. Gong F, Liu G, Zhai X, Zhou J, Cai Z, Li Y: Quantitative analysis of an engineered CO2fixing Escherichia coli reveals great potential of heterotrophic $\mathrm{CO} 2$ fixation. Biotechnol Biofuels 2015, 8:86.

38. Li YH, Ou-Yang FY, Yang CH, Li SY: The coupling of glycolysis and the Rubisco-based pathway through the non-oxidative pentose phosphate pathway to achieve low carbon dioxide emission fermentation. Bioresour Technol 2015, 187:189-197.

39. Guadalupe-Medina V, Wisselink HW, Luttik MA, de Hulster E, Daran JM, Pronk JT, van Maris AJ: Carbon dioxide fixation by Calvin-Cycle enzymes improves ethanol yield in yeast. Biotechnol Biofuels 2013, 6:125.

40. Abubackar HN, Veiga MC, Kennes C: Carbon monoxide fermentation to ethanol by Clostridium autoethanogenum in a bioreactor with no accumulation of acetic acid. Bioresour Technol 2015, 186:122-127.

41. Kopke M, Held C, Hujer S, Liesegang H, Wiezer A, Wollherr A, Ehrenreich A, Liebl W, Gottschalk G, Durre P: Clostridium ljungdahlii represents a microbial production platform based on syngas. Proc Natl Acad Sci U S A 2010, 107:13087-13092.

42. Fei Q, Guarnieri MT, Tao L, Laurens LM, Dowe N, Pienkos PT: Bioconversion of natural gas to liquid fuel: opportunities and challenges. Biotechnol Adv 2014, 32:596-614.

43. Haynes CA, Gonzalez R: Rethinking biological activation of methane and conversion to liquid fuels. Nat Chem Biol 2014, 10:331-339.

44. Whitaker WB, Jones JA, Bennett K, Gonzalez JE, Vernacchio VR, Collins SM, Palmer MA, Schmidt S, Antoniewicz MR, Koffas MA, et al.: Engineering the Biological Conversion of Methanol to Specialty Chemicals in Escherichia coli. Metab Eng 2016.

45. Lessmeier L, Pfeifenschneider J, Carnicer M, Heux S, Portais JC, Wendisch VF: Production of carbon-13-labeled cadaverine by engineered Corynebacterium glutamicum using carbon-13-labeled methanol as co-substrate. Appl Microbiol Biotechnol 2015, 99:10163-10176.

46. Jorda J, Suarez C, Carnicer M, ten Pierick A, Heijnen JJ, van Gulik W, Ferrer P, Albiol J, Wahl A: Glucose-methanol co-utilization in Pichia pastoris studied by metabolomics and instationary (1)(3)C flux analysis. BMC Syst Biol 2013, 7:17.

47. Gebreselassie NA, Antoniewicz MR: (13)C-metabolic flux analysis of co-cultures: A novel approach. Metab Eng 2015, 31:132-139.

48. Antoniewicz MR: Methods and advances in metabolic flux analysis: a mini-review. $J$ Ind Microbiol Biotechnol 2015, 42:317-325.

49. Iwatani S, Van Dien S, Shimbo K, Kubota K, Kageyama N, Iwahata D, Miyano H, Hirayama K, Usuda Y, Shimizu K, et al.: Determination of metabolic flux changes during fed- 
batch cultivation from measurements of intracellular amino acids by LC-MS/MS. $J$ Biotechnol 2007, 128:93-111.

50. Antoniewicz MR: Tandem mass spectrometry for measuring stable-isotope labeling. Curr Opin Biotechnol 2013, 24:48-53.

51. McCloskey D, Young JD, Xu S, Palsson BO, Feist AM: MID Max: LC-MS/MS Method for Measuring the Precursor and Product Mass Isotopomer Distributions of Metabolic Intermediates and Cofactors for Metabolic Flux Analysis Applications. Anal Chem 2016, 88:1362-1370.

52. McConnell BO, Antoniewicz MR: Measuring the Composition and Stable-Isotope Labeling of Algal Biomass Carbohydrates via Gas Chromatography/Mass Spectrometry. Anal Chem 2016, 88:4624-4628.

53. Antoniewicz MR, Kelleher JK, Stephanopoulos G: Measuring deuterium enrichment of glucose hydrogen atoms by gas chromatography/mass spectrometry. Anal Chem 2011, 83:3211-3216.

54. Choi J, Grossbach MT, Antoniewicz MR: Measuring complete isotopomer distribution of aspartate using gas chromatography/tandem mass spectrometry. Anal Chem 2012, 84:4628-4632.

55. Long CP, Antoniewicz MR: Metabolic flux analysis of Escherichia coli knockouts: lessons from the Keio collection and future outlook. Curr Opin Biotechnol 2014, 28:127-133.

*56. Khodayari A, Zomorrodi AR, Liao JC, Maranas CD: A kinetic model of Escherichia coli core metabolism satisfying multiple sets of mutant flux data. Metab Eng 2014, 25:5062.

This paper describes a detailed ensemble kinetic model of $E$. coli core metabolism that encompasses 138 reactions, 93 metabolites and 60 substrate-level regulatory interactions. The described model construction pipeline provides a systematic approach for future improvements as more flux data becomes available.

57. Long CP, Gonzalez JE, Sandoval NR, Antoniewicz MR: Characterization of physiological responses to 22 gene knockouts in Escherichia coli central carbon metabolism. Metab Eng 2016, 37:102-113.

**58. Crown SB, Long CP, Antoniewicz MR: Integrated 13C-metabolic flux analysis of 14 parallel labeling experiments in Escherichia coli. Metab Eng 2015, 28:151-158.

This study demonstrates the power of parallel labeling experiments and integrated ${ }^{13} \mathrm{C}$ MFA for high-resolution flux analysis. In this work, 14 parallel labeling experiments were successfully integrated to generate the most accurate and precise flux map measured thus far for E. coli.

59. Antoniewicz MR: Parallel labeling experiments for pathway elucidation and 13C metabolic flux analysis. Curr Opin Biotechnol 2015, 36:91-97.

60. Au J, Choi J, Jones SW, Venkataramanan KP, Antoniewicz MR: Parallel labeling experiments validate Clostridium acetobutylicum metabolic network model for $\mathbf{C}$ metabolic flux analysis. Metab Eng 2014, 26:23-33. 
61. Cordova LT, Antoniewicz MR: (13)C metabolic flux analysis of the extremely thermophilic, fast growing, xylose-utilizing Geobacillus strain LC300. Metab Eng 2016, 33:148-157.

62. Crown SB, Marze N, Antoniewicz MR: Catabolism of Branched Chain Amino Acids Contributes Significantly to Synthesis of Odd-Chain and Even-Chain Fatty Acids in 3T3-L1 Adipocytes. PLoS One 2015, 10:e0145850.

63. Ahn WS, Antoniewicz MR: Parallel labeling experiments with [1,2-(13)C]glucose and [U(13)C]glutamine provide new insights into CHO cell metabolism. Metab Eng 2013, 15:34-47.

64. Ahn WS, Crown SB, Antoniewicz MR: Evidence for transketolase-like TKTL1 flux in CHO cells based on parallel labeling experiments and (13)C-metabolic flux analysis. Metab Eng 2016, 37:72-78.

65. Crown SB, Kelleher JK, Rouf R, Muoio DM, Antoniewicz M: Comprehensive metabolic modeling of multiple 13C-isotopomer data sets to study metabolism in perfused working hearts. Am J Physiol Heart Circ Physiol 2016:ajpheart 0042802016.

66. Crown SB, Antoniewicz MR: Selection of tracers for 13C-metabolic flux analysis using elementary metabolite units (EMU) basis vector methodology. Metab Eng 2012, 14:150-161.

*67. Crown SB, Long CP, Antoniewicz MR: Optimal tracers for parallel labeling experiments and $13 \mathrm{C}$ metabolic flux analysis: A new precision and synergy scoring system. Metab Eng 2016, 38:10-18.

This is the most comprehensive study performed so far focused on optimal design of labeling experiments for ${ }^{13} \mathrm{C}-\mathrm{MFA}$. The study demonstrates that flux precision can be dramatically improved when multiple tracers are used in parallel labeling experiments.

68. David H, Krogh AM, Roca C, Akesson M, Nielsen J: CreA influences the metabolic fluxes of Aspergillus nidulas during growth on glucose and xylose. Microbiol 2005, 151:2209-2221.

69. Grotkjaer T, Christakopoulos P, Nielsen J, Olsson L: Comparative metabolic network analysis of two xylose fermenting recombinant Saccharomyces cerevisiae strains. Metab Eng 2005, 7:437-444.

70. Long CP, Au J, Gonzalez JE, Antoniewicz MR: 13C metabolic flux analysis of microbial and mammalian systems is enhanced with GC-MS measurements of glycogen and RNA labeling. Metab Eng 2016, 38:65-72.

71. Fonseca C, Neves AR, Antunes AM, Noronha JP, Hahn-Hagerdal B, Santos H, SpencerMartins I: Use of in vivo $13 \mathrm{C}$ nuclear magnetic resonance spectroscopy to elucidate L-arabinose metabolism in yeasts. Appl Environ Microbiol 2008, 74:1845-1855.

72. Fendt SM, Sauer U: Transcriptional regulation of respiration in yeast metabolizing differently repressive carbon substrates. BMC Syst Biol 2010, 4:12.

73. Matheron $C$, Delort AM, Gaudet G, Forano E: In vivo 13C NMR study of glucose and cellobiose metabolism by four cellulolytic strains of the genus Fibrobacter. Biodegradation 1998, 9:451-461.

74. Benisch F, Boles E: The bacterial Entner-Doudoroff pathway does not replace glycolysis in Saccharomyces cerevisiae due to the lack of activity of iron-sulfur cluster enzyme 6-phosphogluconate dehydratase. J Biotechnol 2014, 171:45-55. 
75. Klingner A, Bartsch A, Dogs M, Wagner-Dobler I, Jahn D, Simon M, Brinkhoff T, Becker J, Wittmann C: Large-Scale 13C flux profiling reveals conservation of the EntnerDoudoroff pathway as a glycolytic strategy among marine bacteria that use glucose. Appl Environ Microbiol 2015, 81:2408-2422.

76. Xiong W, Lee TC, Rommelfanger S, Gjersing E, Cano M, Maness PC, Ghirardi M, Yu J: Phosphoketolase pathway contributes to carbon metabolism in cyanobacteria. Nature Plants 2016:doi:10.1038/nplants.2015.1187.

77. Guo W, He R, Ma L, Jia W, Li D, Chen S: Construction of a constitutively expressed homo-fermentative pathway in Lactobacillus brevis. Appl Microbiol Biotechnol 2014, 98:6641-6650.

78. Gonzalez-Rodriguez I, Gaspar P, Sanchez B, Gueimonde M, Margolles A, Neves AR: Catabolism of glucose and lactose in Bifidobacterium animalis subsp. lactis, studied by 13C Nuclear Magnetic Resonance. Appl Environ Microbiol 2013, 79:7628-7638.

79. Young JD, Shastri AA, Stephanopoulos G, Morgan JA: Mapping photoautotrophic metabolism with isotopically nonstationary (13)C flux analysis. Metab Eng 2011, 13:656-665.

80. Russmayer H, Buchetics M, Gruber C, Valli M, Grillitsch K, Modarres G, Guerrasio R, Klavins K, Neubauer S, Drexler H, et al.: Systems-level organization of yeast methylotrophic lifestyle. BMC Biol 2015, 13:80.

81. Reaser BC, Yang S, Fitz BD, Parsons BA, Lidstrom ME, Synovec RE: Non-targeted determination of (13)C-labeling in the Methylobacterium extorquens AM1 metabolome using the two-dimensional mass cluster method and principal component analysis. J Chromatogr A 2016, 1432:111-121.

82. Bogorad IW, Chen CT, Theisen MK, Wu TY, Schlenz AR, Lam AT, Liao JC: Building carbon-carbon bonds using a biocatalytic methanol condensation cycle. Proc Natl Acad Sci U S A 2014, 111:15928-15933.

83. Siegel JB, Smith AL, Poust S, Wargacki AJ, Bar-Even A, Louw C, Shen BW, Eiben CB, Tran HM, Noor E, et al.: Computational protein design enables a novel one-carbon assimilation pathway. Proc Natl Acad Sci U S A 2015, 112:3704-3709. 


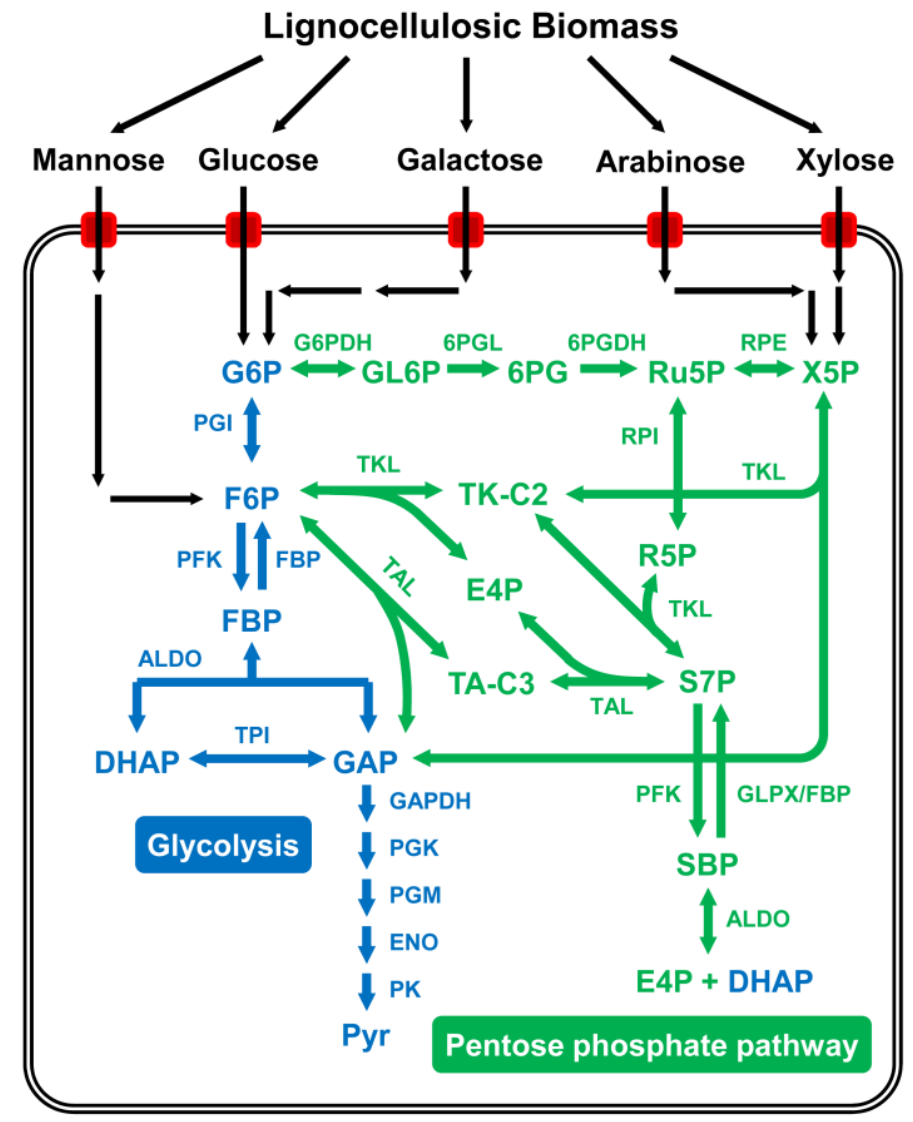




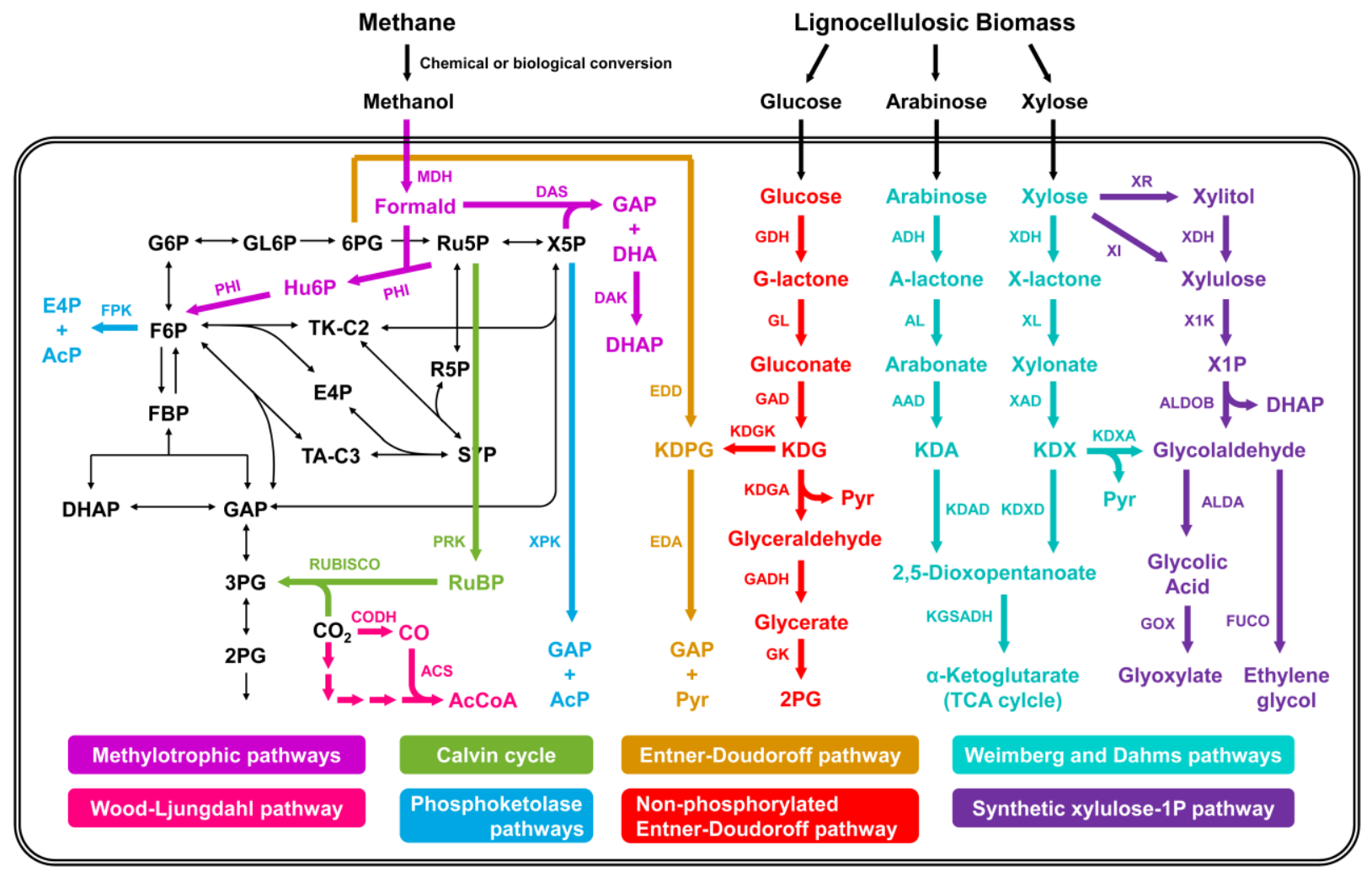


A Isotopic Labeling Experiments

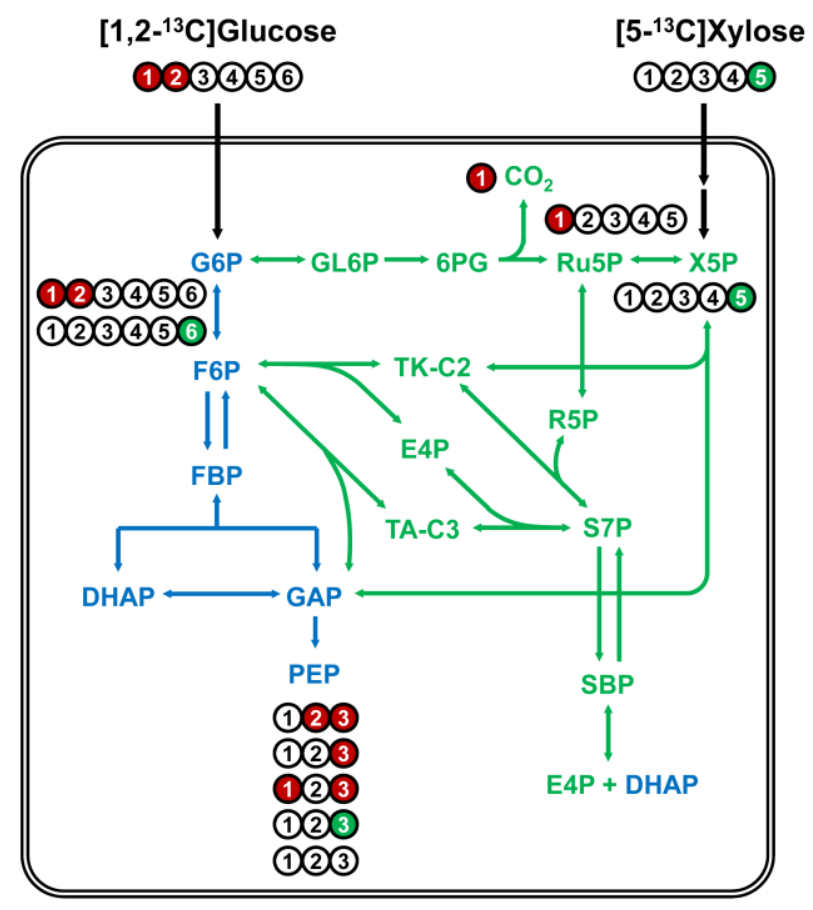

B Isotopic Labeling Measurements

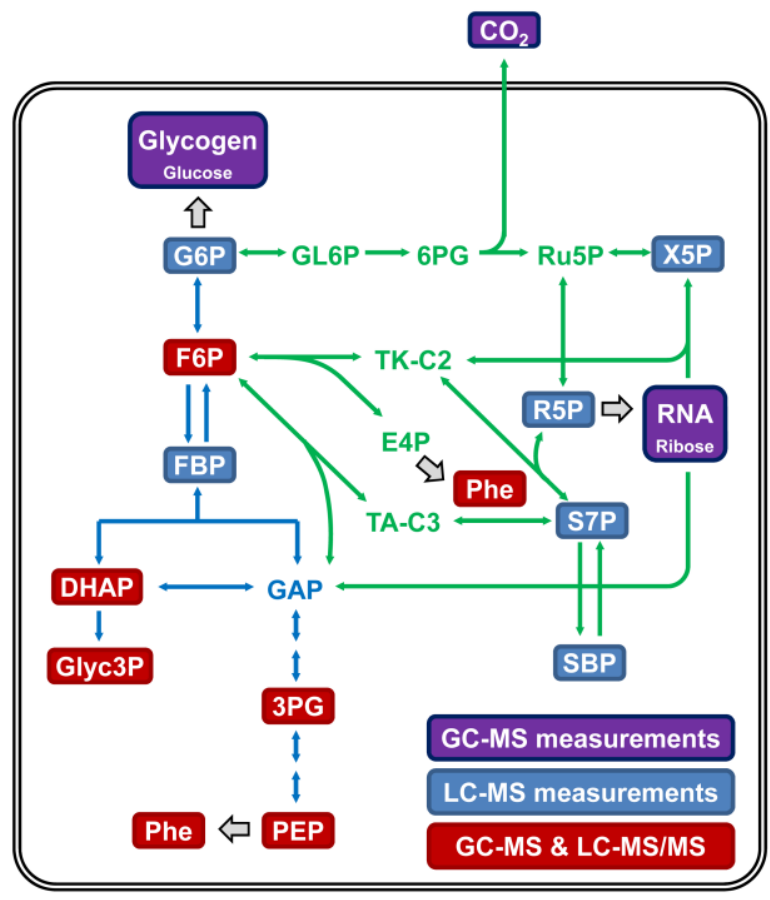


Traditional ${ }^{13} \mathrm{C}$-MFA

(single tracer) or (multiple tracers)
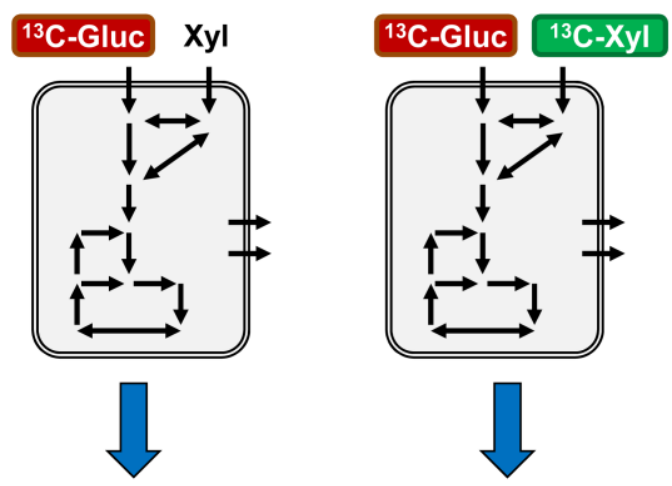

${ }^{13}$ C-MFA

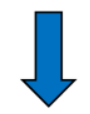

Flux Map



Flux Map



vs. Parallel labeling experiments

(single tracers in parallel experiments)

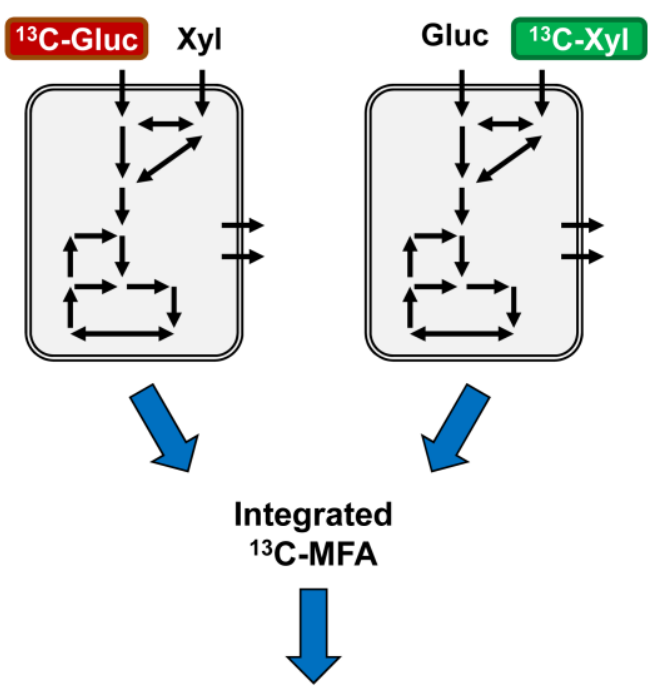

Flux Map

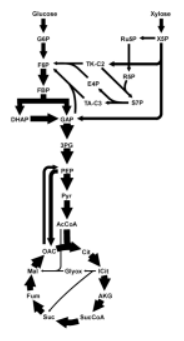




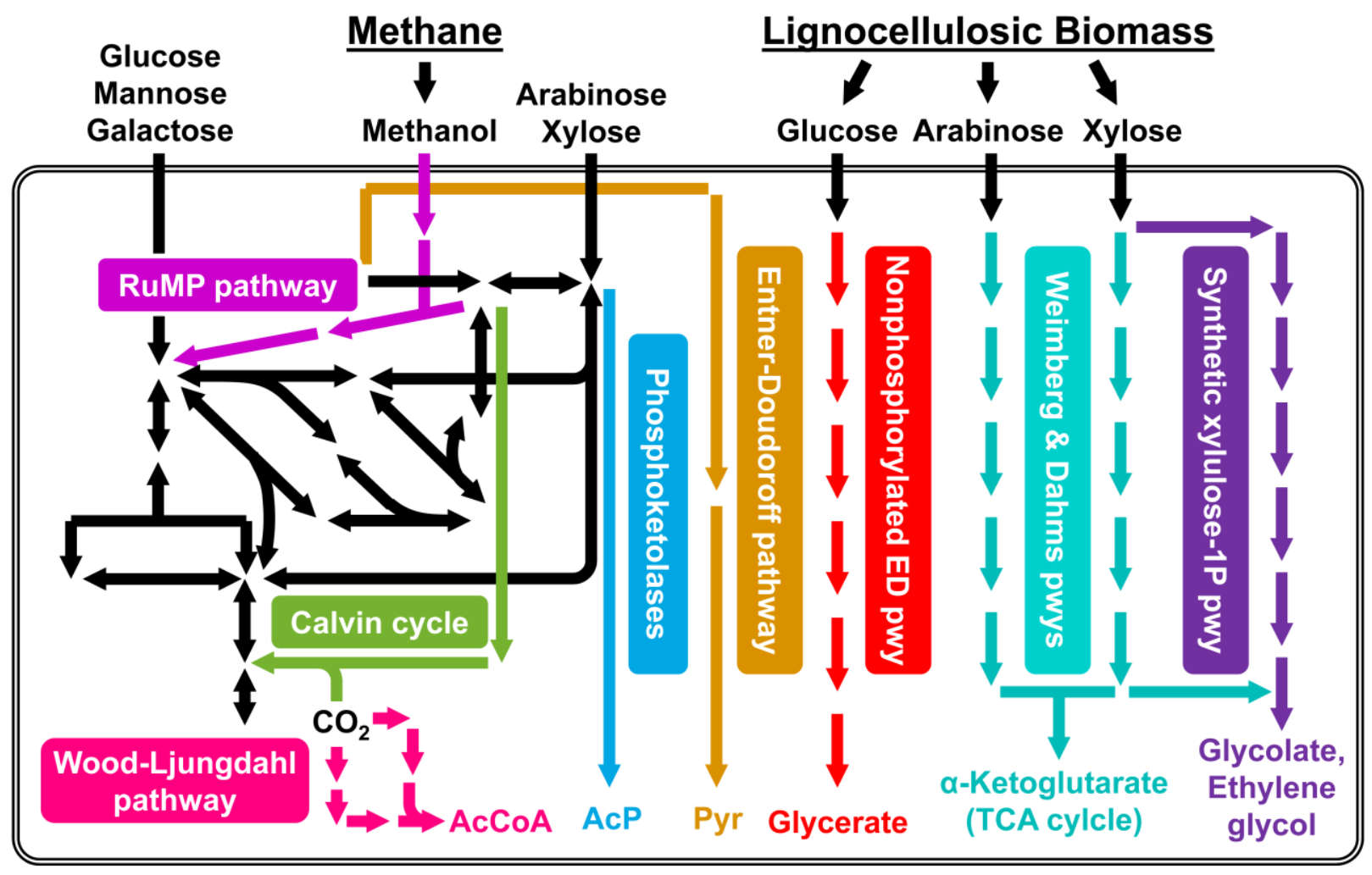

